\title{
EL POLIMORFISMO DE LA /S/ EXPLOSIVA EN EL NOROESTE DE MÉXICO
}

El español de México se caracteriza por su fuerte consonantismo y por la preferencia general a la consonante sobre la vocal ${ }^{1}$. Quimogramas del habla verifican la desaparición de vocales y llevan a dos fonólogos a proponer la existencia de consonantes silábicas en el español mexicano².

1 "Entre otros hispanohablantes, un mexicano se reconoce por su entonación... por su tendencia a alargar la articulación de /s/ y por una preferencia general por la consonante a la vocal", D. Lincoln CANFIELD, Spanish pronunciation in the Americas, University of Chicago Press, Chicago, 1981, p. 60. Para estudios fonológicos sobre el español de México, véanse $\mathrm{CH}$. Carroll Marden, "La fonología del español en la ciudad de Méjico", $B D H, 4$ (1938), 87-187; VíCTOR M. SuÁrez, El español que se habla en Yucatán: apuntamientos filológicos, Díaz Massa, Mérida, 1945; J. MatLuck, "La pronunciación del español en el Valle de México", NRFH, 6 (1951), 109-120; H. V. KING, "Outline of Mexican Spanish phonology", SL, 10 (1952), 5162; Juan M. Lope Blanch, "Estado actual del español en México", Presente y futuro de la lengua española, Oficina Internacional de Información y Observación del Español, Madrid, 1964, pp. 79-91; D. Cárdenas, "The Spanish of Jalisco", PMLA, 70 (1955), 556-561; P. V. Cassano, "Mexican Spanish /-s/ in relation to Aztec influence", $S L, 15$ (1975), 55-61; Peter Boyd-Bowman, El habla de Guanajuato, UNAM, México, 1960; Giorgio Sabino Antonio Presissinotto, Fonología del español hablado en la Ciudad de México. Ensayo de un método sociolingüistico, El Colegio de México, México, 1975; R. Ávila, "Realizaciones tensas de /s/ en la ciudad de México", $A L M, 11$ (1973), 125-139; J. López ChÁvez, "El fonema /s/ en el habla de La Cruz, Sinaloa", NRFH, 16 (1977), 332-340; BeATriz Garza Cuarón, El español hablado en la ciudad de Oaxaca, México (Caracterización fonética y léxica), El Colegio de México, México, 1987; y Juan M. Lope Blanch, Atlas lingüístico de México, El Colegio de México, México, 1991.

2 "A manera de conclusiones... con las salvedades necesarias, nos atrevemos a hablar de consonantes silábicas en el español mexicano", M. J. Canellada de Zamora y A. Zamora Vicente, "Vocales caducas en el español mexicano", $N R F H, 14$ (1960), 221-241, cita p. 240. "Es evidente que la 
Otro rasgo distintivo del español de México es la articulación prolongada de $/ \mathrm{s} / 3$. Este proceso lingüístico puede interpretarse como una función compensatoria. El tiempo perdido en la caída de la vocal se recupera en el alargamiento de la consonante.

$\mathrm{Al}$ mismo tiempo que se identifican estas tendencias generales en la producción de la sibilante/s/ en México, se reconoce otro fenómeno importante, su polimorfismo.

El polimorfismo se define como "la concurrencia de dos o más formas lingüísticas - ya sean fonéticas, gramaticales o léxicas- que alternan libremente para desempeñar una misma función" ". El hecho de ser/s/ el fonema más frecuente entre los consonánticos hace destacar su variabilidad ${ }^{5}$. Los aspectos particulares de /s/ y el polimorfismo que exhibe la $s$ implosiva sobre todo, han sido el tema de muchos estudios específicos ${ }^{6}$. Además,

pérdida de las vocales resulta al oído español peninsular el fenómeno más detonante y más curioso de todo el español americano... el fenómeno existe en todas las clases sociales... Por otra parte, no existe en la conciencia lingüística de los hablantes la menor sanción contraria a la práctica de esa síncopa violenta... Esto nos prueba que el fenómeno es considerado normal dentro de la conciencia lingüística de los hablantes y se halla en terreno abonado para su propagación y fijación', p. 225.

3. CANField, op. cit.; J. C. Zamora MunNÉ, "Las zonas dialectales del español americano", BANLE, 4/5 (1979-1980), 57-67. Una zona dialectal, la núm. 2, se describe así: “México, excepto la costa oriental y las regiones limítrofes con Guatemala. -s final + [ = conservada]. Conservación consonántica. . . articulación ápicodental tensa de $/ \mathrm{s} / \ldots$..."

"JUAN M. LOPE BLANCH, "En torno al polimorfismo", Investigaciones sobre dialectología mexicana, UNAM, México, 1990, pp. 7-33, cita p. 7.

5 “El fonema más frecuente entre los consonánticos es /s/, que en nuestro recuento ocupa $8.00 \%$ (N[avarro Tomás] 8.50, Z[ipf y Rogers] 8.12)", Emilio Alarcos Llorach, Fonología española, Gredos, Madrid, 1961, p. 13.

${ }^{6}$ D. Alonso, "Sobre la -s final de sílaba en el mundo hispánico", $E L H$, t. 1, pp. 47-53; Diana R. Sekraoui, Change and compensation: Parallel reakening of $/ s /$ in Italian, French and Spanish, Peter Lang, New York, 1989; A. H. OBaid, "The vagaries of the Spanish 's' ", H, 56 (1973), 60-67; T. TerRELL, "La aspiración en el español de Cuba. Observaciones teóricas", RLA, 13 (1975), 97-107; T. Terrell, "Final /s/ in Cuban Spanish", H, 62 (1979), 599-612; Orlando Albas, Estratificación social del español de Santiago de los Caballeros: la /s/implosiva, Tesis, Universidad de Puerto Rico, Río Piedras, 1982; J. M. LipsKi, "Reducción de /s/ en el español de Honduras", NRFH, 32 (1983), 171-188; H. Cedergren, "En torno a la variación de la $s$ final de sílaba en Panamá: análisis cuantitativo", en Corrientes actuales en la dialectología del Caribe hispánico: Actas de un simposio, ed. H. López Morales, Universidad de Puerto Rico, Río Piedras, 1978, pp. 36-50; Rocío Caravedo, Estudios sobre el español de Lima, t. 1: Variación contextual de la sibilante, Pontificia Universidad Católica del Perú, Lima, 1983; H. Valdivieso, G. Tassara, J. Magaña 
las variantes aspiradas $[\mathrm{x}] /[\mathrm{h}]$ igual que la omisión del sonido [ø], son rasgos distintivos que sirven para identificar diferentes zonas dialectales ${ }^{7}$. En México, con la excepción de ciertas zonas costales y del sur, la aspiración y la deleción no se consideran características $^{8}$.

Concedidas las tendencias hacia la conservación y el alargamiento de /s/ en el español hablado en México, es natural que se despierte la curiosidad cuando uno observa fenómenos contrarios, es decir, la pérdida, el relajamiento y la aspiración de /s/ seguida de una vocal ( $s$ explosiva). Observemos las variantes de /s/ en el habla de dos personas mayores de un pueblo serrano del estado de Sonora:

(Un señor mayor, consultante $\mathrm{F}$ ): - $\mathrm{Y}$ un día por allá mil nove[s]ientos quin[s]e por el ocho o die[ $\left.{ }^{z}\right]$ de di[s]iembre, pa[s]ó Pancho Villa para acá venía de la derrota de Agua Prieta y de Hermo[s]illo, ¿no? Y cuatro generale $[\mathrm{s}]$ con $[\mathrm{s}] \mathrm{u}\left[{ }^{\mathrm{s}}\right]$ tropa $\left[{ }^{\mathrm{s}}\right]$ llegaron aquí a la ca[s]a, al corral, ¿no? [s]e portaron muy bien con no[s]otro[s]. Ca[s]i le puedo de[h]ir que dema[s]iado caballero[s]o[s]. Lo mi[z]mo Pancho Villa. A nadie le hi[s]o nada. Él tenía... tomaba la palabra y le[h] echaba a lo[s] gringo[s], a[s]í de[h]ía él, por lo que le hi[șieron en Columbo, ¿ve?

(Una señora mayor, consultante A): $-\mathrm{Y}$ yo todo el tiempo viví aquí y era raro que [s]e fueran allá y que [s]e me cayeran allí al agua, y é $\left[^{\mathrm{s}}\right] \operatorname{ro}\left[^{\mathrm{s}}\right]$ no[s] [s]alen. No má[s] ven el agua y allí [s]e van, no[s] llevamo[s] tra[ $\left[{ }^{z}\right]$ de ello[s $\left[{ }^{\mathrm{s}}\right]$ no má $\left[{ }^{\mathrm{s}}\right]$.

Quieren... [s]í. Peligro[s]o. [h]í, pue[s]. Y aquí no. Y luego abajo iba el río. Corría el río con mucha agua. Con mucha agua. En

y C. Duque, "Le /s/ implosif dans l'espagnol de Concepción et de Valparaíso [Chile]", LingP, 24 (1988), 131-141; N. E. Donni de Mirande, "El segmento fonológico/s/ en el español de Rosario [Argentina]", LEA, 11 (1989), 89-115; J. M. LIPSKI, "On the reduction of /s/ in Philippine Creole Spanish: Implications for historical Spanish dialectology", Diachronica: International Journal for Historical Linguistics/Revue Internationale pour la Linguistique Historique, 3 (1986), 43-66; J. M. LIPSKI, "On the loss of/s/ in 'Black' Spanish", N, 70 (1986), 208-216.

${ }^{7} \mathrm{La}-s$ final, las realizaciones de $j, g e, g i$ y el voseo son los tres rasgos distintivos escogidos por J. C. Zamora MunNé en "Las zonas dialectales del español americano". Véase también Melvyn C. Resnick, Phonological variants and dialect identification in Latin 'American Spanish, Mouton, The Hague, 1975.

${ }^{8}$ Zamora Munné, art. cit., p. 63. 
e[s]e tiempo corría allá abajo. Mucha agua allí y no, gra[s]ia[h] a Dio[s], y aquí vivía con ello[s] chiquito[ø] y no me pa[s]aba nada.

(La misma señora, imitando a un hijo suyo): -Y luego e[s]e mío, grandote. Por e[s]o hi[ $\left.{ }^{\mathrm{s}}\right] \mathrm{o}^{-}$cuando hi["]ieron... allí e[s]a puerta porque [s]e daba a cada rato andaba agachando aquí pegaba con e[s]o de acá allí. Y a cada rato andaba di[s]iendo: -A[s]í vamo[h] a ha[s]er e[h]a puerta alta, di[s]e, por no andarme agachando tanto.

¿Cómo se explican estas realizaciones de /s/ explosiva - [í] 'sí', [é ha puér tal 'esa puerta', [grá sıa ha diós] 'gracias a Dios' - escuchadas en un pueblo de Sonora? ¿Cómo se compaginan la aspiración [h] y la elisión [ $\emptyset]$ con la norma mexicana del consonantismo fuerte y de la /s/ alargada?

Otros aspectos de estos fenómenos surgen también al contemplar la variación polimórlica de /s/ inicial de sílaba: su extensión, su frecuencia, la distribución de los alófonos según los diferentes contextos y su uso por hombres y mujeres.

Para investigar las circunstancias que rigen en el uso alofónico de /s/ y para cuantificar su distribución en una parte del Noroeste de México, se emprendió un estudio del habla en Sahuaripa, Sonora, un pueblo de la Sierra Madre Occidental.

Sahuaripa interesa por su fundación antigua (1631) y por su

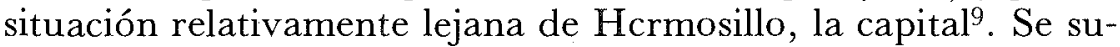
pone que un municipio serrano colindante con el estado de Chihuahua ofrece un contexto lingüístico con menos tendencias niveladoras que las que se encuentran en la capital del estado o en pueblos más cercanos a ella ${ }^{10}$.

El corpus consiste en conversaciones individuales e informales grabadas con diez personas, cinco mujeres y cinco hombres. Las entrevistas varían en su duración. La transcripción más extensa ocupa 34 hojas escritas a máquina a doble espacio (consultante C), las más breves, 3 hojas (consultantes I, J). La discrepancia

9 "'[Sahuaripa] fue fundada el año de 1631 por los misioneros de la Compañía de Jesús con el nombre de Nuestra Señora de los Ángeles de Sahuaripa", Francisco R. Almada, Diccionario de historia, geografía y biografía sonorenses, Gobierno del Estado de Sonora, Hermosillo, 1983, p. 618.

${ }^{10}$ Para una discusión de la nivelación que ocurre en Hermosillo, consúltese M. T. Alessi y A. L. Torres, "Aspectos fonéticos del español sonorense", ponencia dada en el Primer Encuentro sobre la Lingüistica en el. Noroeste de Sonora, Hermosillo, Sonora, 25 octubre de 1990. La Memoria del Encuentro está en prensa. 
en el número de casos de /s/ analizados para cada persona se debe principalmente a la mayor disponibilidad de las mujeres y el acceso a ellas de parte de una investigadora foránea.

Las personas consultadas tienen tres aspectos en común: 1) generación lingüística (edad mínima de 55 años) ${ }^{11}$; 2) lugar de nacimiento (el municipio de Sahuaripa); y 3 ) residencia (toda o casi toda su vida en el mismo lugar).

Los consultantes se designan por mayúsculas: las femeninas A, B, C, D y E; los masculinos F, G, H, I, J. El cuadro que se da a continuación incluye información biográfica pertinente para cada consultante: lugar de nacimiento, estado civil, oficio y escolaridad. "Texto" se refiere al número de hojas que forman el corpus; la última columna contiene el número total de alófonos de /s/ analizados en cada entrevista.

Cuadro 1

\begin{tabular}{lcllrc}
\hline Nacimiento & Estado civil & Oficio & Escolaridad & Texto & Núm. de/s/ \\
\hline A Nácori Chico & casada & ama de casa & media & 10 & 1099 \\
B Arivechi & soltera & tortillera & poca & 4 & 164 \\
C Sahuaripa & viuda & partera & analfabeta & 34 & 398 \\
D Sahuaripa & casada & ama de casa & media & 17 & 777 \\
E Nácori Chico & casada & ama de casa & primaria & 10 & 777 \\
F Sahuaripa & casado & propietario & superior & 27 & 420 \\
G El Tigre de & viudo & cultivador de & poca & 6 & 180 \\
$\quad$ Corodepe & & güérigos & & & \\
H Arivechi (?) & casado & propietario, ex- & secunda- & 15 & 256 \\
& & gambucino & ria (?) & & \\
I Tiópare & \multirow{2}{*}{ soltero } & vaquero retirado & poca & 3 & 90 \\
J Sahuaripa & casado & jornalero & poca & 3 & 50 \\
\hline
\end{tabular}

Las conversaciones rinden el total de 4211 ejemplos de /s/. Un poco más de la mitad (2 243) son casos de /s/ explosiva, es

${ }^{11}$ Se ha seguido la división de edad según el Cuestionario para el estudio coordinado de la norma lingüistica culta de las principales ciudades de Iberoamérica y de la Peninsula Ibérica. I: Fonética y fonología, C.S.I.C., Madrid, 1973, p. xv. También véase Juan M. Lope Blanch, El habla de la Ciudad de México. Materiales para su estudio, UNAM, México, 1971, p. 6: "Los informantes serán hombres y mujeres, en una distribución proporcional de $50 \%$ aproximadamente. Se eligirán representantes de tres generaciones, de acuerdo con la siguiente distribución; a) de 25 a 35 años $=30 \% ; b$ ) de 36 a 55 años $=45 \% ; c$ ) de más de 55 años $=25 \%$ ". 
decir, $s$ que inicia una sílaba. Estos 2243 ejemplos forman la documentación para este estudio.

Los contextos de /s/ explosiva se dividen en dos partes: 1) sseguida por una vocal después de pausa de cualquier duración (\# sV) y después de consonante $(\mathrm{CsV})$; 2) -s- intervocálica sea medial o final de palabra $(\mathrm{VsV})$.

Los restantes 1968 casos son de /s/ implosiva, es decir, $s$ que termina una sílaba: 3) -s final antes de pausa (Vs \#) y 4) $-s$ antes de consonante (sG). Las cifras para /s/ implosiva se encuentran en el Apéndice (tablas 7-12). El Apéndice también contiene tablas en que se dan los números de casos y los porcentajes para todos los ejemplos de /s/ implosiva y explosiva encontrados en el corpus (tablas 13-15).

Seis alófonos además de la elisión $[\emptyset]$ se perciben en las grabaciones de los diez sahuaripenses estudiados. Son:

1. [s] predorsoalveolar fricativo sordo;

2. [ $\left.{ }^{\mathrm{s}}\right]$ predorsoalveolar fricativo sordo debilitado;

3. [h] glotal fricativo sordo;

4. [z] predorsoalveolar fricativo sonoro;

5. [ $\mathrm{z}]$ predorsoalveolar fricativo sonoro debilitado;

6. $[\theta]$ interdental fricativo sordo;

7. $[\emptyset]$ elisión.

De las siete realizaciones de /s/ identificadas en el corpus, sólo cinco se encuentran en posición explosiva: $[\mathrm{s}],[\mathrm{s}],[\mathrm{h}],[\theta]$ y elisión [Ø].

Las tablas 1-6 contienen datos sobre la frecuencia y distribución de los alófonos de /s/ explosiva. La información se presenta en el orden siguiente: primero se da la estadística para los consultantes juntos y para los dos grupos separados por sexo. Después se dan los números de casos y porcentajes de uso alofónico individual, una tabla para las consultantes femeninas y otra tabla para los consultantes masculinos.

En la tabla 1 se analiza la totalidad de casos de la $s$ - inicial de sílaba después de una pausa de cualquier duración (\#sV) y después de una consonante ( $\mathrm{CsV}$ ). Allí se ve que predomina con mucho el alófono [s]. Casi nueve veces de diez, el $87.21 \%$, cuando los sahuaripenses mayores articulan una $s$ inicial, sale [s].

A pesar de este predominio de [s], dos tendencias llaman la atención: 1) las mujeres aspiran $s$-inicial casi dos veces más que los hombres $\left(8.13 \%\right.$ a $\left.4.70 \%{ }^{*}\right)$; 2) los hombres omiten el sonido ocho veces más que las mujeres $\left(4.70 \%{ }^{*}\right.$ a $\left..52 \% \%^{*}\right)$. 
TABLA 1

/s/ explosiva

s- inicial después de pausa y después de consonante

\begin{tabular}{|c|c|c|c|c|c|}
\hline Todos los casos 727 & $=100.00 \%$ & Fem. & $=578$ & Masc. & $=149$ \\
\hline [s] & $87.21 \% *$ & 505 & $87.37 \%$ & 129 & $86.58 \%$ * \\
\hline [h] & $7.43 \% *$ & 47 & $8.13 \%$ & 7 & $4.70 \% *$ \\
\hline$[\mathrm{s}]$ & $3.85 \%$ & 23 & $3.98 \%$ * & 5 & $3.35 \%$ \\
\hline$[\emptyset]$ & $1.37 \%$ & 3 & $.52 \%{ }^{*}$ & 7 & $4.70 \%^{*}$ \\
\hline$[\theta]$ & $.14 \% *$ & 0 & 0 & 1 & $.67 \%$ \\
\hline Total & $100.00 \%$ & & $100.00 \%$ & & $100.00 \%$ \\
\hline
\end{tabular}

* Todas las cifras señaladas con un asterisco han sido redondeadas por medio del aumento de un céntimo de un porcentaje.

En la tabla 2, se observa que dos mujeres, A y D, exhiben cuatro variaciones polimórficas para $s$ - inicial de sílaba. La consultante $\mathrm{C}$, al contrario, no varía en su realización de $s$ - como [s]. Otra persona, E, varía menos de uno por ciento $\left(.79 \%^{*}\right)$ en su articulación [s]. A pesar de que la consultante B no omite el sonido, ella alterna entre [s] más de la mitad del tiempo y [ $\left.{ }^{\mathrm{s}}\right]$.

TABLA 2

s- inicial después de pausa y después de consonante Consultantes femeninas

\begin{tabular}{lccccc}
\hline Núm. de & $A$ & $B$ & $C$ & $D$ & $E$ \\
casos & $205=100 \%$ & $31=100 \%$ & $55=100 \%$ & $160=100 \%$ & $127=100 \%$ \\
\hline$[\mathrm{s}]$ & $84.88 \%^{*}$ & $58.07 \%^{*}$ & $100.00 \%$ & $82.50 \%$ & $99.21 \%$ \\
{$[\mathrm{~s}]$} & $4.39 \%$ & $35.48 \%$ & 0 & $1.25 \%$ & $.79 \% *$ \\
{$[\mathrm{~h}]$} & $9.76 \%^{*}$ & $6.45 \%$ & 0 & $15.63 \% *$ & 0 \\
{$[\varnothing]$} & $.97 \% *$ & 0 & 0 & $.62 \%$ & 0 \\
& $100.00 \%$ & $100.00 \%$ & $100.00 \%$ & $100.00 \%$ & $100.00 \%$ \\
\hline
\end{tabular}

Menos consultantes masculinos (tabla 3) manifiestan polimorfismo para $s$ - inicial que sus contrapartes femeninas. Tres de los hombres (F, H, J) usan [s] un poco más del $90.00 \%$. Los consultantes G e I la articulan [s] unas siete veces de cada diez. G e I también relajan el sonido; éste más que aquél $\left(14.29 \%{ }^{*}\right.$ a $\left.8.11 \%^{*}\right)$. Dos $(\mathrm{G}$ y J) lo aspiran; tres $(\mathrm{F}, \mathrm{H}, \mathrm{I})$ lo eliden. $\mathrm{G}$ revela un uso infrecuente e idiosincrático $(2.70 \%)$ al realizar $s$ - inicial como interdental $[\theta]$. De los cinco hombres observados, es $G$ 
quien exhibe mayor polimorfismo. Aunque $\mathrm{G}$ no omite $s^{-}$, tiende a aspirar el sonido más que ningún otro consultante masculino o femenino (16.22\%*). Los consultantes F y J presentan un contraste interesante. Las pocas veces que $\mathrm{J}$ no articula [s], él lo aspira $(7.14 \%)$, pero no lo omite; $\mathrm{F}$ lo omite $(5.08 \%)$, pero no lo aspira.

TABLA 3

s- inicial después de pausa y después de consonante Consultantes masculinos

\begin{tabular}{|c|c|c|c|c|c|}
\hline $\begin{array}{l}\text { Núm. de } \\
\text { casos }\end{array}$ & $\begin{array}{c}F \\
59=100 \%\end{array}$ & $\begin{array}{c}G \\
31=100 \%\end{array}$ & $\begin{array}{c}H \\
25=100 \%\end{array}$ & $\begin{array}{l}I \\
14=100 \%\end{array}$ & $\begin{array}{c}J \\
14=100 \%\end{array}$ \\
\hline$[\mathrm{s}]$ & $94.92 \% *$ & $72.97 \%$ * & $92.00 \%$ & $71.42 \%$ & $92.86 \% *$ \\
\hline$[\mathrm{s}]$ & 0 & $8.11 \% *$ & 0 & $14.29 \% *$ & 0 \\
\hline$[\mathrm{h}]$ & 0 & $16.22 \% *$ & 0 & 0 & $7.14 \%$ \\
\hline$[\phi]$ & $5.08 \%$ & 0 & $8.00 \%$ & $14.29 \% *$ & 0 \\
\hline \multirow[t]{2}{*}{ [V] } & 0 & $2.70 \%$ & 0 & 0 & 0 \\
\hline & $100.00 \%$ & $100.00 \%$ & $100.00 \%$ & $100.00 \%$ & $100.00 \%$ \\
\hline
\end{tabular}

La tabla 4 revela más altos porcentajes de variantes alofónicas, o sea, mayor grado de polimorfismo para la -s-intervocálica del que se observa para la $s$ - inicial. Sigue prevaleciendo [s]. Cuatro veces de cinco $(80.85 \%)$, las mujeres producen el sonido predorsoalveolar sordo fricativo. Falta poco para que los hombres alcancen la misma frecuencia $(77.10 \% *)$. Cuando no producen el sonido predorsoalveolar, los consultantes como grupo alternan entre la aspiración [h] y el relajamiento [s] con aproximadamente la misma frecuencia $(7.92 \%$ * y $7.39 \%$ * respectivamente). También se observa la elisión del sonido (3.69\%), pero mucho menos que $[\mathrm{h}]$ y [s].

El $1.12 \%$ de ocurrencias del alófono interdental [ $\theta$ ] se debe principalmente al idiolecto de un consultante masculino, $\mathrm{H}$ (véase la tabla 6).

Es interesante señalar dos tendencias particulares. Aunque la elisión de /s/ explosiva ocurre relativamente poco en el habla sahuaripense, las mujeres la eliden más que los hombres cuando es intervocálica ( $4.54 \%$ a $1.27 \%$ ). Los hombres tienden a omitirla más cuando es inicial después de una pausa y después de una consonante $(4.70 \% *$ a $.52 \%$ * tabla 1$)$.

La tabla 5 revela que todas las mujeres emplean 4 alófonos cuando /s/ es intervocálica. Así es que en el habla femenina, el 
TABLA 4

-s- intervocálica medial y entre vocablos

\begin{tabular}{|c|c|c|c|}
\hline \multicolumn{2}{|c|}{1.516 casos $=100.00 \%$} & \multirow{2}{*}{$\frac{\text { Fem. } 1123=100.00 \%}{908=80.85 \% *}$} & \multirow{2}{*}{$\frac{\text { Masc. } 393=100.00 \%}{303=77.10 \% *}$} \\
\hline$[\mathrm{s}]$ & $79.88 \%$ & & \\
\hline [h] & $7.92 \% *$ & $84=7.48 \% *$ & $36=9.16 \%$ \\
\hline$[s]$ & $7.39 \% *$ & $80=7.13 \% *$ & $32=8.14 \%$ \\
\hline$[\emptyset]$ & $3.69 \% *$ & $51=4.54 \%$ & $5=1.27 \%$ \\
\hline \multirow[t]{2}{*}[\theta]{} & $1.12 \%$ & $0 \quad 0$ & $17=4.33 \%^{*}$ \\
\hline & $100.00 \%$ & $100.00 \%$ & $100.00 \%$ \\
\hline
\end{tabular}

fonema /s/ muestra más polimorfismo en la posición intervocálica que en posición inicial después de pausa y después de consonante.

Al mismo tiempo, se observan diferencias individuales en la distribución alofónica, sobre todo en el idiolecto de la consultante B. Ésta realiza -s- como [s] más de la mitad de las veces $(58.33 \%)$, pero también recurre a la aspiración [h] $(18.33 \%)$ y a la forma relajada $\left.{ }^{[}\right]\left(16.67 \%{ }^{*}\right)$ mucho más que las cuatro otras mujeres.

Las consultantes A, C y D articulan el alófono predorsoalveolar fricativo sordo [s] más de 4 veces de cada $5(83.48 \%$ *, $86.54 \%$, $\left.82.84 \%{ }^{*}\right)$; E lo articula así unas 3 veces de $4\left(76.52 \%{ }^{*}\right)$. De ahí que se vea la oposición entre el uso regular de [s] por A, C, D y E y el uso mediano (58.33\%) de B.

TABLA 5

-s- intervocálica medial y entre vocablos

Consultantes femeninas

\begin{tabular}{lcccccc}
\hline Núm. de & $A$ & $B$ & $C$ & $D$ & $E$ \\
casos & $357=100 \%$ & $60=100 \%$ & $156=100 \%$ & $303=100 \%$ & $247=100 \%$ \\
\hline$[\mathrm{s}]$ & $83.48 \% *$ & $58.33 \%$ & $86.54 \% *$ & $82.84 \% *$ & $76.52 \% *$ \\
{$[\mathrm{~s}]$} & $8.40 \%$ & $16.67 \% *$ & $5.13 \% *$ & $3.96 \%$ & $8.10 \% *$ \\
{$[\mathrm{~h}]$} & $7.00 \%$ & $18.33 \%$ & $5.13 \% *$ & $11.22 \%$ & $12.95 \%$ \\
{$[\emptyset]$} & $1.12 \%$ & $6.67 \% *$ & $3.20 \%$ & $1.98 \%$ & $2.43 \% *$ \\
& $100.00 \%$ & $100.00 \%$ & $100.00 \%$ & $100.00 \%$ & $100.00 \%$ \\
\hline
\end{tabular}

La tabla 6 contiene los porcentajes de $-s$ - de parte de los 5 hombres estudiados. Hay que examinar el uso individual para comprender bien la distribución. 
El alófono interdental $[\theta]$ forma parte del inventario de sonidos de solamente dos de los consultantes masculinos $(\mathrm{F} \mathrm{y} \mathrm{H})$ y principalmente de H. Éste lo articula más de una vez de 10 (el $11.67 \% *$ ) mientras que el consultante $\mathrm{F}$ realiza $[\theta]$ sólo el $1.70 \%$ * Estos dos son los únicos de los 10 consultantes grabados que realizan /s/ intervocálica como interdental. Esta particularidad distorsiona un poco las cifras.

TABLA 6

-s- intervocálica medial y entre vocablos

Consultantes masculinos

\begin{tabular}{|c|c|c|c|c|c|}
\hline $\begin{array}{l}\text { Núm. de } \\
\text { casos }\end{array}$ & $\begin{array}{c}F \\
15=100 \%\end{array}$ & $\begin{array}{c}G \\
117=100 \%\end{array}$ & $\begin{array}{c}H \\
61=100 \%\end{array}$ & $\begin{array}{c}I \\
120=100 \%\end{array}$ & $20=100 \%$ \\
\hline$[\mathrm{s}]$ & $77.40 \%$ & $73.77 \%$ & $80.00 \%$ & $90.00 \%$ & $46.67 \%$ * \\
\hline$\left[{ }^{s}\right]$ & $10.17 \% *$ & $16.39 \%$ & $.83 \%$ & $5.00 \%$ & $13.33 \%$ \\
\hline [h] & $9.03 \%$ & $8.20 \%$ * & $6.67 \%^{*}$ & $5.00 \%$ & $40.00 \%$ \\
\hline$[\emptyset]$ & $1.70 \% *$ & $1.64 \%$ * & $.83 \%$ & 0 & 0 \\
\hline \multirow[t]{2}{*}[\theta]{} & $1.70 \%$ * & 0 & $11.67 \% *$ & 0 & \\
\hline & $100.00 \%$ & $100.00 \%$ & $100.00 \%$ & $100.00 \%$ & $100.00 \%$ \\
\hline
\end{tabular}

Salta a la vista en las seis tablas el hecho de que cuatro variantes principales y la deleción componen las realizaciones alofónicas de /s/ en posición libre (explosiva). ¿A qué se debe tanta variación?

Una parte de la explicación viene de la naturaleza de un sonido sibilante. En un estudio de /s/ en el habla de niños hispanoparlantes, un investigador afirma que la variedad de /s/ es de esperarse a causa de los problemas que surgen en la producción, transmisión y percepción de las sibilantes ${ }^{12}$. Según él, la producción de éstas es múltiple porque requiere una configuración lingual precisa para crear la forma de canal indicada para cada articulación. A su vez, tal configuración afecta las percepciones acústicas del oyente. El mismo investigador propone que /s/ puede ser inherentemente variable en los dialectos españoles.

Otra investigadora mantiene que "los seis reflejos diferentes de /s/ documentados en las lenguas romances son todos el resul-

12 K. W. MASON, "Child language and ohter evidence for /s/ variation in Spanish dialects", Papers in Applied Linguistics, 3 (1987), 64-78. 
tado de la economía articulatoria lograda por medio de la reducción del movimiento articulatorio, el cual se hace o por asimilación o por facilitar transiciones entre sonidos o por deleción"'13.

Hay que recordar que lo que distingue el fonema fricativo y sordo /s/ de otros sonidos fricativos sordos como /f/ y / $/$ /, es la estrechez del canal por el cual corre el aire expulsado de los pulmones y la formación del surco o canal. La flexibilidad de la lengua le permite tomar muchas posiciones: puede crear una superficie cóncava $[\mathrm{s}$ ] o una forma convexa $[\mathrm{s}]$. Al mismo tiempo la lengua tiene la capacidad de moderar la estrechez del canal. Por un lado, si la lengua se hace tensa y si aprieta mucho contra la parte inmóvil, el sonido resultante es marcadamente sibilante y tenso [s]. Por otro lado, si la lengua no establece contacto firme y decisivo con la parte inmóvil, en otras palabras, si se relaja, ocurre el fenómeno contrario, el debilitamiento. El sonido producido es débil y relajado [s]. La posición de la lengua es baja $[\perp]$.

Simultáneamente con los movimientos descritos, varias secciones de la lengua pueden usarse para producir el surco: el ápice, el predorso, el dorso, el posdorso. Con tal de que se respete la fricción, el lugar en donde se produce /s/ puede variar sin cambiar su naturaleza sibilante.

La relativa velocidad del discurso afecta no sólo la manera en que el hablante articula los sonidos sino también la manera en que el interlocutor los percibe. En general, cuanto más se acelere la velocidad de emisión, tanto mayor es el relajamiento articulatorio y, paralelamente, menos precisa la percepción acústica.

En el español americano, el fonema /s/ suele articularse con la lengua contra los dientes [ș] o en contacto con la región dentoalveolar [s]. Lo que uno observa en las variantes de /s/ en el habla de Sahuaripa, Sonora, es un cambio en el lugar de articulación desde una posición anterior de la cavidad bucal a una posición posterior.

Para ilustrar esto, se conceptualiza el perfil de la boca como una línea horizontal y el espacio entre el articulador y el lugar de articulación como una línea vertical.

En el eje horizontal, los labios se encuentran a la izquierda y la glotis a la derecha. Al colocar los alófonos sahuaripenses sobre este continuo, al extremo izquierdo, está el sonido interden-

${ }^{13}$ Diana R. Seklaoui, op. cit., p. 13. 
tal $[\theta]$; más a la derecha, el sonido dentoalveolar [s]; más a la derecha aún, el sonido glotal [h]; y al otro extremo, a mano derecha, el cero.

Los cambios en el grado de tensión muscular del articulador (la lengua) se registran en el eje vertical. Abajo está la ausencia de tensión, el cero [Ø]; más alto está [h] en el cual hay tensión en la región supraglotal; más arriba se encuentra [s], sonido relajado, con la lengua baja; hacia arriba está [s] articulado con mayor tensión y con menos espacio entre el articulador y el lugar de contacto; y para completar la analogía, en la cumbre de esta línea vertical está $[\theta]$, sonido interdental.

Así es que el polimorfismo de /s/ explosiva que existe en los sahuaripenses estudiados se debe a dos cambios fonéticos: uno en el lugar de articulación desde la parte anterior de la cavidad bucal hacia la parte posterior; otro en el grado de tensión muscular de la lengua.

Estos dos cambios se ilustran en el uso polimórfico de [s] y [h]. Los dos son alófonos fricativos y sordos. [s] es predorsoalveolar y [h] es glotal. Cuando los sahuaripenses articulan [nohotros], el lugar de articulación para /s/ retrocede a la región supraglotal. El cambio ocurre por el eje horizontal.

Cuando los consultantes dicen [bamos], relajan la consonante final. Ellos reducen el grado de cierre entre la lengua y el área dentoalveolar. Se crea más espacio entre el articulador y los alvéolos. Al bajarse la lengua, ésta ejerce menos esfuerzo en la producción de este alófono [s] que en [s] o en [s:] prolongada.

Si el proceso lingüístico de reducción de esfuerzo explica en parte el polimorfismo de /s/ encontrado en el habla sahuaripense, otro proceso, la disimilación, puede explicar las variaciones encontradas en 'gracias a Dios' gra[s]ia[h]a Dio[s], 'diecisiete' die[h]ils]iete, 'los conocí' lo[h]cono[s]li, 'las encías' la/h] en[s]íals], 'las necesitaba' la[h] ne[sle[s]itaba, 'no más hacen' no málh] a/s]en, etc. Se alternan [s], [h] y [s] evitando de esta manera la repetición de tres [s] seguidas. Es posible que sea ésta una alternativa intuitivamente empleada.

También conviene señalar el hecho de que al sustituir [h] por [s] en [no-hó-tros] o en [no-hó-troh], el sahuaripense no causa ambigüedad o equívoco para su interlocutor. En español no compiten léxicamente el vocablo nosotros y *nojotros o*nojotroj, ni escuela y * ejcuela, ni tres meses y *tej meses, ni uno se fue y uno *je fue, ni hace $\mathrm{y}^{*}$ haje, etc. Aun cuando existen dos vocablos, uno con [s] y otro con $[\mathrm{h}]$, como, por ejemplo, la afirmación sí y la interjec- 
ción ijí!, los contextos en que aparecen no son similares. El reemplazo de [s] por [h] no suele producir equívocos de significado en el interlocutor.

Otro factor contribuye a la impresión exagerada de que los serranos aspiran mucho la $s$ : la aspiración se encuentra en expresiones comunes de alta frecuencia en el uso diario. Expresiones ya citadas como, por ejemplo, 'gracia [h] a Dios', 'má[h] o menos', la afirmación ' $/ h / i$ ' (y ' $i$ ), formas del adjetivo demostrativo 'e[h] $e^{\prime}$, los pronombres ' $n o[h] o t r o h '$ y 'IJhe' ('se'), la locución verbal 'vamo[h] $a$ ' y otros usos como 'de [h]arauripa' surgen frecuentemente en la conversación. Aunque la estadística no confirma un alto porcentaje de aspiración para la /s/ explosiva en general (el $7.74 \%$ ), el hecho de que [h] y no [s] aparezca en expresiones corrientes crea la impresión de que, efectivamente, el alófono aspirado es de uso regular. Además, hay que considerar la posibilidad de que, como observa Seklaoui, "la aspiración [en español] en posición inicial no sea tanto un proceso fonológico como un proceso léxico, prevaleciente sobre todo en ciertas palabras: $s i$, señor, señora, señorita y el pronombre reflexivo $s e^{\text {" } 14}$. La problemática aquí consiste en el peso que se le da a la determinación fonológica contra la determinación léxica. En mi opinión, pesan más los procesos fonológicos (especialmente el relajamiento del articulador y el retroceso del lugar de articulación) que lo que pesa la influencia léxica.

Viene al caso comparar los resultados del presente análisis del habla de Sahuaripa, Sonora, con otro estudio del español hablado en el pueblo de La Cruz, Sinaloa, el estado al sur de Sonora ${ }^{15}$. A base de datos sacados de 7 personas ( 3 mujeres, 4 hombres), el investigador encuentra que "el fonema/s/ en La Cruz, en general, es sumamente polimórfico: se produce predorsoalveolar, dentalizado, aspirado, etc."'16. Su análisis lo lleva a concluir que 1) la aspiración $[\mathrm{h}]$ es un fenómeno propio del habla masculina de los jóvenes (más que de las mujeres y más de los jóvenes que de los viejos); 2) la aspiración de -s-intervocálica es tan fre-

${ }^{14}$ Ibid., p. 52

${ }^{15}$ J. López Chávez, "El fonema/s/ en el habla de La Cruz, Sinaloa". Este estudio forma parte del proyecto de "Delimitación de las Zonas Dialectales" de El Colegio de México.

${ }^{16}$ Ibid., pp. 334-335. 
cuente como la aspiración de $-s$ final ${ }^{17}$. Además, este investigador clasifica el fenómeno como relativamente moderno ${ }^{18}$.

El estudio del habla sahuaripense varía del de La Cruz en que tanto las mujeres como los hombres aspiran /s/ en todos los contextos, los hombres un poco más (8.43\%) que las mujeres $\left(7.53 \%^{*}\right)^{19}$. Intervocálicamente, la aspiración se encuentra el $7.91 \% *$. Cuando $/ \mathrm{s} /$ es final antes de pausa, se aspira el $4.08 \% *$ y cuando $/ \mathrm{s} /$ viene antes de consonante, hay aspiración el $7.09 \%$ de los $\operatorname{casos}^{20}$. La aspiración de /s/ explosiva se compara más con $s$ seguida de consonante que con $s$ final seguida de pausa. En cuanto a la relativa modernidad del fenómeno, es difícil comparar los dos lugares. En Sahuaripa, sólo se escogió a personas de más de 55 años de edad; en La Cruz, las personas entrevistadas son de tres generaciones.

Para concluir, se puede decir que el estudio sobre la $s$ explosi-

17 Ibid., pp. 335-337.

${ }^{18}$ Ibid., p. 338. También el estudio de Rocío Caravedo ( $o p$. cit.) puede servir de punto de comparación. El análisis de Caravedo interesa aquí porque el Perú comparte con México el rasgo característico de la conservación de $s$. Los dos análisis se semejan en que 1) las dos investigadoras hacían el papel de observadora-participante; 2) los datos proceden de entrevistas informales y no de cuestionarios; y 3) la mitad de los consultantes son hombres y la otra mitad son mujeres. Las diferencias entre el estudio limeño y el sahuaripense son: 1) Lima es una metrópoli, Sahuaripa, un pueblo; 2) hay 12 consultantes de Lima, 10 de Sahuaripa; 3) en el estudio limeño, se recoge media hora de conversación de cada consultante, la duración de las entrevistas en Sahuaripa varía; 4) los limeños representan 3 generaciones, los sahuripenses, una sola generación, la mayor; 5) el nivel educacional de los limeños es alto, profesional, en Sahuaripa el nivel es bajo y mediano; 6) para Lima, se dividen los contextos de/s/ en 3:a) SC; $b$ ) SV;c) S \#, para Sahuaripa, se dividen los contextos en 4:a) \#SV/CSV; b) VSV; c) SC; d) S\#; 7) el corpus de Lima rinde 12938 ejemplos, el de Sahuaripa, 4211 ejemplos. Consciente de las diferen. cias entre los dos estudios y especialmente del número superior de ejemplos de Lima, se resumen los datos para /s/:

\begin{tabular}{lcrrr}
\hline & Lima $(p .94)$ & \multicolumn{1}{c}{ Sahuaripa } \\
\hline Retención & {$[\mathrm{s}]$} & $10085=77.94 \%$ & {$[\mathrm{~s}]$} & $2565=60.91 \%$ \\
Aspiración & {$[\mathrm{h}]$} & $1673=13.00 \%$ & {$[\mathrm{~h}]$} & $326=7.74 \%$ \\
Deleción & $\phi$ & $611=4.72 \%$ & $\phi$ & $276=6.56 \%$ \\
Sonorización & {$[\mathrm{z}]$} & $137=1.05 \%$ & {$[\mathrm{z}]$} & $45=1.07 \%$ \\
Debilitamiento & {$\left[{ }^{2}\right]$} & $422=3.26 \%$ & {$\left[{ }^{\mathrm{s}}\right]$} & $889=21.11 \%$ \\
Debilitamiento & & 0 & {$\left[{ }^{\mathrm{z}}\right]$} & $91=2.16 \%$ \\
Interdentalización & & 0 & {$[\theta]$} & $19=0.45 \%$ \\
Total & $12928=100.00 \%$ & & $4211=100.00 \%$ \\
\hline
\end{tabular}

${ }^{19}$ Véase la tabla 13 en el Apéndice.

${ }^{20}$ Véanse las tablas 7 y 10 en el Apéndice. 
va en un pueblo serrano de Sonora demuestra el predominio del alófono [s] sobre los otros alófonos. Al mismo tiempo se puede ver que la /s/ explosiva es polimórfica: tiene 4 otras maneras de representarse en el habla de los sahuaripenses mayores de edad además de $[\mathrm{s}]$ : $[\mathrm{s}],[\mathrm{h}],[\emptyset]$ y $[\theta]$ en el idiolecto de unos pocos. Tanto las mujeres como los hombres exhiben usos polimórficos $\mathrm{de} / \mathrm{s} /$ explosiva en el habla informal. No cabe duda de que el polimorfismo está vivo entre los sahuaripenses mayores.

DOLORES BROWN

Profesora Emérita,

University of Arizona

\begin{abstract}
APÉNDICE
Se observa en las tablas 7-9 que los sahuaripenses articulan [s] más que sus contrapartes femeninas. Éstas relajan el sonido más que aquéllos. Los dos grupos omiten la consonante moderadamente. La deleción de $-s$ es notablemente más frecuente que la aspiración.

En este contexto se observa una distribución polimórfica de [s], [h] y [ [] más pareja respecto a las consultantes femeninas (del $26 \%$ al $32 \%$ ) y menos pareja en cuanto a los masculinos (del $17 \%$ al $50 \%$ ).
\end{abstract}

TABLA 7

-s \# final antes de una pausa de cualquier duración

Todos los consultantes

\begin{tabular}{ccrc}
\hline 515 casos $=100.00 \%$ & Fern. $426=100.00 \%$ & Masc. $89=100.00 \%$ \\
\hline$[\mathrm{s}]$ & $32.43 \% *$ & $122=28.64 \% *$ & $45=50.56 \%$ \\
{$\left[^{\mathrm{s}}\right]$} & $31.40 \% *$ & $138=32.40 \% *$ & $24=26.97 \% *$ \\
{$[\phi]$} & $25.24 \%$ & $114=26.76 \%$ & $16=17.98 \% *$ \\
{$\left[^{\mathrm{z}}\right]$} & $5.44 \% *$ & $28=6.57 \%$ & 0 \\
{$[\mathrm{~h}]$} & $4.08 \% *$ & $17=3.99 \%$ & $4=4.49 \%$ \\
{$[\mathrm{z}]$} & $1.16 \%$ & $6=1.41 \% *$ & 0 \\
{$[\theta]$} & $.19 \% *$ & $1=.23 \%$ & 0 \\
& $100.00 \%$ & $100.00 \%$ & $100.00 \%$ \\
\hline
\end{tabular}


TABLA 8

-s \# final antes de una pausa de cualquier duración Consultantes femeninas

\begin{tabular}{|c|c|c|c|c|c|}
\hline $\begin{array}{l}\text { Num. de } \\
\text { casos }\end{array}$ & $\begin{array}{c}A \\
114=100 \%\end{array}$ & $\begin{array}{c}B \\
28=100 \%\end{array}$ & $\begin{array}{c}C \\
64=100 \%\end{array}$ & $\begin{array}{c}D \\
56=100 \%\end{array}$ & $\begin{array}{c}E \\
164=100 \%\end{array}$ \\
\hline [s] & $47.37 \% *$ & $3.57 \%$ & $17.19 \%$ * & $58.93 \% *$ & $14.02 \%$ \\
\hline$\left[{ }^{s}\right]$ & $30.70 \%$ & $35.71 \%$ & $31.25 \%$ & $35.71 \%$ & $32.32 \% *$ \\
\hline [h] & $.88 \% *$ & $10.72 \%{ }^{*}$ & $1.56 \%$ & $5.36 \%$ * & $5.49 \% *$ \\
\hline$[\phi]$ & $21.05 \%$ & $50.00 \%$ & $50.00 \%$ & 0 & $26.83 \%$ * \\
\hline$[\mathrm{z}]$ & 0 & 0 & 0 & 0 & $3.66 \%$ * \\
\hline$\left[{ }^{2}\right]$ & 0 & 0 & 0 & 0 & $17.07 \%$ \\
\hline \multirow[t]{2}{*}[\theta]{} & 0 & 0 & 0 & 0 & $.61 \%$ * \\
\hline & $100.00 \%$ & $100.00 \%$ & $100.00 \%$ & $100.00 \%$ & $100.00 \%$ \\
\hline
\end{tabular}

TABLA 9

-s \# final antes de una pausa de cualquier duración Consultantes masculinos

\begin{tabular}{lrcccc}
\hline $\begin{array}{l}\text { Núm. de } \\
\text { casos }\end{array}$ & $\begin{array}{c}F \\
25=100 \%\end{array}$ & $\begin{array}{c}G \\
24=100 \%\end{array}$ & $\begin{array}{c}\text { H } \\
15=100 \%\end{array}$ & $\begin{array}{c}I \\
15=100 \%\end{array}$ & $\begin{array}{c}J \\
10=100 \%\end{array}$ \\
\hline$[\mathrm{s}]$ & $68.00 \%$ & $58.33 \%$ & $73.33 \% \%^{*}$ & $20.00 \%$ & 0 \\
{$[\mathrm{~s}]$} & $16.00 \%$ & $16.67 \% *$ & $26.67 \% *$ & $53.33 \%$ & $40.00 \%$ \\
{$[\mathrm{~h}]$} & $4.00 \%$ & $8.33 \%$ & 0 & 0 & $10.00 \%$ \\
{$[\oint]$} & $12.00 \%$ & $16.67 \% *$ & 0 & $26.67 \% *$ & $50.00 \%$ \\
& $100.00 \%$ & $100.00 \%$ & $100.00 \%$ & $100.00 \%$ & $100.00 \%$ \\
\hline
\end{tabular}

En las tablas 10-12 se nota que el alófono relajado [\$] es el más frecuente en el contexto $\mathrm{SC}$ aunque la distribución de [s] y [ $\left.{ }^{\mathrm{s}}\right]$ en el grupo femenino es casi igual. Los hombres aspiran la sibilante seguida por una consonante más que las mujeres. Los dos grupos eliden el sonido con la misma frecuencia. Los porcentajes revelan que en este contexto/s/ se omite y se aspira más de lo que se sonoriza.

TABLA 10

-sc medial y sin pausa entre vocablos

Todos los consultantes

\begin{tabular}{cccc}
\hline 1453 casos $=100.00 \%$ & Fem. $1088=100.00 \%$ & Masc. $365=100.00 \%$ \\
\hline$\left.{ }^{\mathrm{s}}\right]$ & $40.40 \%^{*}$ & $435=39.98 \%$ & $160=43.84 \% \%^{*}$ \\
{$[\mathrm{~s}]$} & $38.06 \%^{*}$ & $427=39.25 \%^{*}$ & $118=32.33 \%^{*}$ \\
{$[\emptyset]$} & $7.43 \%$ & $82=7.53 \%$ & $26=7.12 \%$ \\
{$[\mathrm{~h}]$} & $7.09 \%^{*}$ & $68=6.25 \%$ & $35=9.59 \% *$ \\
{$\left[{ }^{\mathrm{z}}\right]$} & $4.54 \%^{*}$ & $45=4.14 \%^{*}$ & $21=5.75 \%$ \\
{$[\mathrm{z}]$} & $2.48 \%^{*}$ & $31=2.85 \%^{*}$ & $5=1.37 \% *$ \\
& $100.00 \%$ & $100.00 \%$ & $100.00 \%$ \\
\hline
\end{tabular}


TABLA 11

-sc medial y sin pausa entre vocablos Consultantes femeninas

\begin{tabular}{lrcrrr}
\hline Núm. de & $A$ & $B$ & $C$ & $D$ & $E$ \\
casos & $423=100 \%$ & $45=100 \%$ & $123=100 \%$ & $258=100 \%$ & $239=100 \%$ \\
\hline$[\mathrm{s}]$ & $43.50 \%^{*}$ & $15.56 \% *$ & $51.22 \%^{*}$ & $48.06 \%$ & $23.85 \%^{*}$ \\
{$\left[{ }^{\mathrm{s}}\right]$} & $35.70 \%^{*}$ & $44.44 \%$ & $30.08 \%$ & $25.97 \%^{*}$ & $63.60 \%^{*}$ \\
{$[\mathrm{~h}]$} & $2.84 \%^{*}$ & $6.67 \%^{*}$ & $7.32 \%^{*}$ & $12.02 \%^{*}$ & $5.44 \%^{*}$ \\
{$[\phi]$} & $8.04 \%^{*}$ & $20.00 \%$ & $5.69 \%$ & $5.81 \%$ & $7.11 \%$ \\
{$[\mathrm{z}]$} & $4.25 \%$ & $4.44 \%$ & $1.63 \% *$ & $4.65 \%$ & 0 \\
{$\left[{ }^{\mathrm{z}}\right]$} & $5.67 \%$ & $8.89 \% *$ & $4.06 \%$ & $3.49 \% *$ & 0 \\
& $100.00 \%$ & $100.00 \%$ & $100.00 \%$ & $100.00 \%$ & $100.00 \%$ \\
\hline
\end{tabular}

TABLA 12

-sc medial y sin pausa entre vocablos

Consultantes masculinos

\begin{tabular}{|c|c|c|c|c|c|}
\hline $\begin{array}{l}\text { Núm. de } \\
\text { casos }\end{array}$ & $\begin{array}{c}F \\
159=100 \%\end{array}$ & $\begin{array}{c}G \\
58=100 \%\end{array}$ & $\begin{array}{c}H \\
96=100 \%\end{array}$ & $\begin{array}{c}I \\
41=100 \%\end{array}$ & $\begin{array}{c}J \\
11=100 \%\end{array}$ \\
\hline$[\mathrm{s}]$ & $39.62 \%$ & $17.24 \%$ & $37.50 \%$ & $21.95 \%$ & 0 \\
\hline$\left[{ }^{8}\right]$ & $39.62 \%$ & $51.73 \%$ * & $41.67 \% *$ & $48.78 \%$ & $63.64 \% *$ \\
\hline [h] & $8.18 \%^{*}$ & $12.07 \%$ * & $9.37 \%$ & $12.20 \% *$ & $9.09 \%$ \\
\hline$[\emptyset]$ & $4.40 \%$ & $5.17 \%$ & $7.29 \%$ & $14.63 \%$ & $27.27 \%$ \\
\hline$[\mathrm{z}]$ & $2.52 \%$ * & $1.72 \%$ & 0 & 0 & 0 \\
\hline \multirow[t]{2}{*}[{}^{z}]{} & $5.66 \%$ & $12.07 \% *$ & $4.17 \% *$ & $2.44 \% *$ & 0 \\
\hline & $100.00 \%$ & $100.00 \%$ & $100.00 \%$ & $100.00 \%$ & $100.00 \%$ \\
\hline
\end{tabular}

En las tablas 13-15 se presenta un panorama global de todos los usos alofónicos de /s/ encontrados en las conversaciones grabadas con los 10 consultantes mayores de Sahuaripa, Sonora. Se agrupan los casos de la /s/ explosiva y de la /s/ implosiva.

De la estadística reunida, se deduce lo siguiente:

1. El alófono [s] se usa 6 veces de 10 en que se articula /s/.

2. ['] se encuentra 2 veces de 10 .

3 . $/ \mathrm{s} /$ se aspira más de lo que se omite.

4. La deleción de /s/ es 2 veces más frecuente que la sonorización, sea ésta fuerte $[z]$ o débil $\left[{ }^{2}\right]$.

5. Los hombres aspiran $/ \mathrm{s} /$ un poco más que las mujeres.

6. Las mujeres eliden $/ \mathrm{s} /$ un poco más que los hombres.

7. Aparece mayor polimorfismo con distribución más pareja entre los alófonos en el contexto $-s$ final (S\#) que en cualquier otro. 
TABLA 13

Resumen de todos los ejemplos de /s/

Todos los consultantes

\begin{tabular}{|c|c|c|c|}
\hline \multicolumn{2}{|c|}{4211 casos $=100.00 \%$} & \multirow{2}{*}{$\frac{\mathrm{Fem} .3215=100.00 \%}{1970=61.27 \%}$} & \multirow{2}{*}{$\frac{\text { Masc. } 996=100.00 \%}{595=59.74 \%^{*}}$} \\
\hline$[\mathrm{s}]$ & $2565=60.91 \%$ & & \\
\hline$[8]$ & $889=21.11 \%$ & $668=20.78 \%^{*}$ & $221=22.19 \%^{*}$ \\
\hline [h] & $326=7.74 \%$ & $242=7.53 \%^{*}$ & $84=8.43 \%$ \\
\hline$[\emptyset]$ & $276=6.56 \% *$ & $224=6.97 \% *$ & $52=5.22 \%$ \\
\hline$[8]$ & $91=2.16 \%$ & $70=2.18 \% *$ & $21=2.11 \%^{*}$ \\
\hline$[\mathrm{z}]$ & $45=1.07 \%^{*}$ & $40=1.24 \%$ & $5=0.50 \%$ \\
\hline \multirow[t]{2}{*}[\theta]{} & $19=0.45 \%$ & $1=0.01 \%^{*}$ & $18=1.81 \%^{*}$ \\
\hline & $100.00 \%$ & $100.00 \%$ & $100.00 \%$ \\
\hline
\end{tabular}

TABLA 14

Resumen de todos los alófonos de $/ s /$

Consultantes femeninas

\begin{tabular}{lccrrr}
\hline $\begin{array}{l}\text { Núm. de } \\
\text { casos }\end{array}$ & $\begin{array}{c}A \\
1099=100 \%\end{array}$ & $\begin{array}{c}B \\
164=100 \%\end{array}$ & $\begin{array}{c}C \\
398=100 \%\end{array}$ & $\begin{array}{c}D \\
777=100 \%\end{array}$ & $\begin{array}{c}E \\
777=100 \%\end{array}$ \\
\hline$[\mathrm{s}]$ & $64.61 \%^{*}$ & $37.20 \%^{*}$ & $66.33 \%$ & $69.50 \%^{*}$ & $50.84 \%^{*}$ \\
{$[\mathrm{~s}]$} & $20.47 \%^{*}$ & $31.10 \%^{*}$ & $16.33 \%$ & $13.00 \%^{*}$ & $29.09 \%^{*}$ \\
{$[\mathrm{~h}]$} & $5.28 \%^{*}$ & $11.58 \%$ & $4.52 \%$ & $11.97 \%^{*}$ & $6.95 \%^{*}$ \\
{$[\phi]$} & $5.82 \%^{*}$ & $16.46 \%$ & $11.06 \%^{*}$ & $2.83 \%$ & $8.62 \%$ \\
{$[\mathrm{z}]$} & $1.64 \%^{*}$ & $1.22 \%^{*}$ & $0.50 \%^{*}$ & $1.54 \%$ & $0.77 \%$ \\
{$[\mathrm{z}]$} & $2.18 \%^{*}$ & $2.44 \%^{*}$ & $1.26 \%^{*}$ & $1.16 \%^{*}$ & $3.60 \%$ \\
{$[\theta]$} & 0 & 0 & 0 & 0 & $0.13 \%^{*}$ \\
& $100.00 \%$ & $100.00 \%$ & $100.00 \%$ & $100.00 \%$ & $100.00 \%$ \\
\hline
\end{tabular}

TABLA 15

Resumen de todos los alófonos de /s/

Consultantes masculinos

\begin{tabular}{lrrrrr}
\hline $\begin{array}{l}\text { Num. de } \\
\text { casos }\end{array}$ & $\begin{array}{c}F \\
420=100 \%\end{array}$ & $\begin{array}{c}G \\
180=100 \%\end{array}$ & $\begin{array}{c}\text { H } \\
256=100 \%\end{array}$ & $\begin{array}{c}I \\
90=100 \%\end{array}$ & $50=100 \%$ \\
\hline$[\mathrm{s}]$ & $65.00 \%$ & $53.33 \%$ & $64.84 \%$ & $44.44 \%$ & $40.00 \%$ \\
{$[\mathrm{~s}]$} & $20.24 \%^{*}$ & $26.11 \%$ & $17.58 \% *$ & $34.45 \%^{*}$ & $26.00 \%$ \\
{$[\mathrm{~h}]$} & $7.14 \%$ & $11.11 \%$ & $6.64 \%$ & $8.89 \%^{*}$ & $18.00 \%$ \\
{$[\phi]$} & $3.81 \%$ & $4.44 \%$ & $3.91 \%$ & $11.11 \%$ & $16.00 \%$ \\
{$[\mathrm{z}]$} & $0.95 \%$ & $0.56 \% *$ & 0 & 0 & 0 \\
{$\left[{ }^{\mathrm{z}}\right]$} & $2.14 \%$ & $3.89 \%^{*}$ & $1.56 \%$ & $1.11 \%$ & 0 \\
{$[\theta]$} & $0.72 \% *$ & $0.56 \%^{*}$ & $5.47 \%^{*}$ & 0 & 0 \\
& $100.00 \%$ & $100.00 \%$ & $100.00 \%$ & $100.00 \%$ & $100.00 \%$ \\
\hline
\end{tabular}

